\title{
Temporal response dynamics of Drosophila olfactory sensory neurons depends on receptor type and response polarity
}

\section{Merid N. Getahun, Dieter Wicher, Bill S. Hansson ${ }^{\dagger}$ and Shannon B. Olsson*t}

Department of Evolutionary Neuroethology, Max Planck Institute for Chemical Ecology, Jena, Germany

\section{Edited by:}

Barry W. Connors, Brown University, USA

\section{Reviewed by:}

Jane L. Witten, University of

Wisconsin-Milwaukee, USA

Alfredo Fontanini, Stony Brook

University, USA

*Correspondence:

Shannon B. Olsson, Department of Evolutionary Neuroethology, Max

Planck Institute for Chemical

Ecology, Hans-Knöll-St. 8,

D-07745 Jena, Germany.

e-mail: solsson@ice.mpg.de

${ }^{t}$ These authors share senior authorship.
Insect olfactory sensory neurons (OSN) express a diverse array of receptors from different protein families, i.e. ionotropic receptors (IR), gustatory receptors (GR) and odorant receptors (OR). It is well known that insects are exposed to a plethora of odor molecules that vary widely in both space and time under turbulent natural conditions. In addition to divergent ligand specificities, these different receptors might also provide an increased range of temporal dynamics and sensitivities for the olfactory system. To test this, we challenged different Drosophila OSNs with both varying stimulus durations (10-2000 ms), and repeated stimulus pulses of key ligands at various frequencies $(1-10 \mathrm{~Hz})$. Our results show that OR-expressing OSNs responded faster and with higher sensitivity to short stimulations as compared to IR- and Gr21a-expressing OSNs. In addition, OR-expressing OSNs could respond to repeated stimulations of excitatory ligands up to $5 \mathrm{~Hz}$, while IR-expressing OSNs required $\sim 5 x$ longer stimulations and/or higher concentrations to respond to similar stimulus durations and frequencies. Nevertheless, IR-expressing OSNs did not exhibit adaptation to longer stimulations, unlike OR- and Gr21a-OSNs. Both ORand IR-expressing OSNs were also unable to resolve repeated pulses of inhibitory ligands as fast as excitatory ligands. These differences were independent of the peri-receptor environment in which the receptors were expressed and suggest that the receptor expressed by a given OSN affects both its sensitivity and its response to transient, intermittent chemical stimuli. OR-expressing OSNs are better at resolving low dose, intermittent stimuli, while IR-expressing OSNs respond more accurately to long-lasting odor pulses. This diversity increases the capacity of the insect olfactory system to respond to the diverse spatiotemporal signals in the natural environment.

Keywords: odorant receptors, ionotropic receptors, pulse resolution, single sensillum recording

\section{INTRODUCTION}

Insect olfactory sensory neurons (OSN) express a large number of receptor proteins of different types. These receptor types include ionotropic receptors (IR), gustatory receptors (GR), and odorant receptors (OR) (Clyne et al., 1999; Vosshall et al., 1999; Benton et al., 2009). IRs are composed of three trans-membrane proteins and co-receptors, while GRs and ORs are seven trans-membrane proteins (Vosshall et al., 1999; Benton et al., 2006, 2009). ORs are co-expressed with the ubiquitous co-receptor Orco, while Gr21a, a $\mathrm{CO}_{2}$ sensor, is co-expressed with Gr63a (Benton et al., 2006; Jones et al., 2007). All OSNs are housed within different morphological types of olfactory hairs, known as sensilla. There appear to be important organizational differences between OSNs that express IRs, GRs, or ORs. Multiple IRs and GRs can be coexpressed per neuron, while OR expression generally follows a one neuron-one receptor rule (Thorne et al., 2004; Wang et al., 2004; Couto et al., 2005; Benton et al., 2009). Receptors from different protein families can also be co-localized in the same sensillum (Couto et al., 2005; Song et al., 2012). For example, in Drosophila, the ab1 sensillum houses four OSNs, three expressing
ORs and one expressing Gr21a. Also, in the Drosophila coeloconic sensillum ac3 an OSN expressing Or35a is co-localized with an OSN expressing Ir75abc (Yao et al., 2005; Silbering et al., 2011).

These diverse receptors have evolved at different points in evolutionary time (Robertson et al., 2003; Croset et al., 2010). Recent research also suggests that many have broad affinity to different chemical classes (Hallem et al., 2004; Yao et al., 2005; Benton et al., 2009; Ai et al., 2010). Yet specificity might not be the only reason for receptor diversification. In the natural environment, insects are constantly challenged with odors not only of diverse molecular types, but with diverse spatio-temporal dynamics. At some distances, odor plumes can present brief and intermittent stimuli (Kaissling et al., 1987; Vickers et al., 2001) with low molecular flux, while at close range or high molecular flux, odors could present a nearly continuous stimulus (Murlis et al., 2000; Louis et al., 2008; Gomez-Marin et al., 2011). These spatiotemporal factors could also be a significant driving force for diversification. The behavior of an insect is a result of the integration of responses from several OSNs expressing a variety of receptor 
types (Silbering et al., 2011). Thus it is worthwhile to characterize the response dynamics across the OSN repertoire.

To address whether these different receptor types exhibit differences in temporal response kinetics, we assess the response dynamics of Drosophila OSNs expressing various receptor types to both different stimulus durations and frequencies. We evaluate the temporal dynamics of antennal OSNs expressing ORs (Or59b and Or35a), IRs (Ir84a, Ir75abc, and Ir41a), and GRs (Gr21a). Or59b-OSNs and Ir41a-OSNs respond with either excitation or inhibition to different ligands, and were chosen to assess the effect of response polarity on temporal kinetics. Or35a- and Ir75abc-OSNs are housed in the same sensillum, and are tested to control for the effects of the perireceptor environment on the temporal response. Finally, Gr21a-expressing OSNs are the only GR-expressing OSNs found on the antenna. Here we show that sensory neurons expressing receptors from different protein families also exhibit different dynamics to brief and intermittent stimuli.

\section{MATERIALS AND METHODS}

Both male and female flies at 2-6 days of age were used. Stocks were maintained on conventional cornmeal agar medium under a $12 \mathrm{~h}$ light: $12 \mathrm{~h}$ dark cycle at $25^{\circ} \mathrm{C}$.

\section{ELECTROPHYSIOLOGY}

A fly was mounted in a cut pipette tip with the head protruding and small amount of wax placed into the tip end to prevent movement. The pipette was then fixed onto a microscope slide with wax and the antennae fixed on a cover slip with a sharpened glass micropipette, similar to (Hallem et al., 2004; Yao et al., 2005; Pellegrino et al., 2010). An electrolytically sharpened tungsten electrode was placed in the eye for grounding and a sharpened tungsten recording electrode was brought into contact with the base of the sensillum using a Luigs and Neumann, SM-59 manipulator (Ratingen, Germany) at $1000 \times$ magnification with an Olympus BX-51 microscope (Olympus Corporation, Tokyo, Japan).

\section{ODOR STIMULI}

Methyl acetate $(>98 \%)$, citral $(>95 \%)$, phenyl acetaldehyde (>90\%), butyric acid (>99\%), 1, 4-diaminobutane and isoamylamine $(>98 \%)$, 1-hexanol $(>99 \%)$, and ethyl hexanoate (>99\%) were purchased from Sigma Aldrich Germany. Phenyl acetaldehyde, methyl acetate, 1-hexanol and ethyl hexanoate were diluted in mineral oil (BioChemika Ultra, Fluka), and butyric acid, 1, 4-diaminobutane, and isoamylamine were dissolved in water. Citral was dissolved in hexane (>99\%, Fluka Analytical, Buchs, Switzerland). We chose odor concentrations within the linear portion of the dose response curve and the tested concentrations are indicated with circles (Figure 1). All concentrations are reported as $\log$ [odor] v/v. For Gr21a stimulation, a $1.5 \mathrm{ml}$ glass vial was filled with pure $\mathrm{CO}_{2}$ and placed into the stimulus system similar to the other stimuli. After each frequency set $(1-10 \mathrm{~Hz})$, the $\mathrm{CO}_{2}$ was refilled.

For frequency stimulation, we used a custom-built multicomponent stimulus system similar to (Olsson et al., 2011). Briefly, $400 \mathrm{ul}$ of appropriate dilutions of each odorant was added

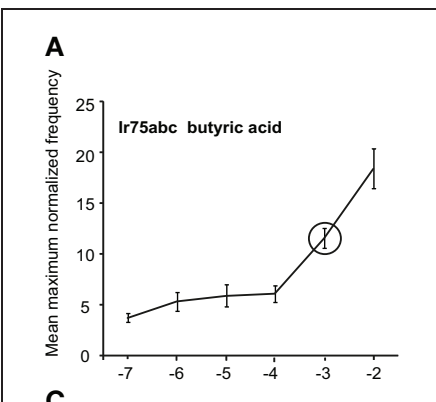

B
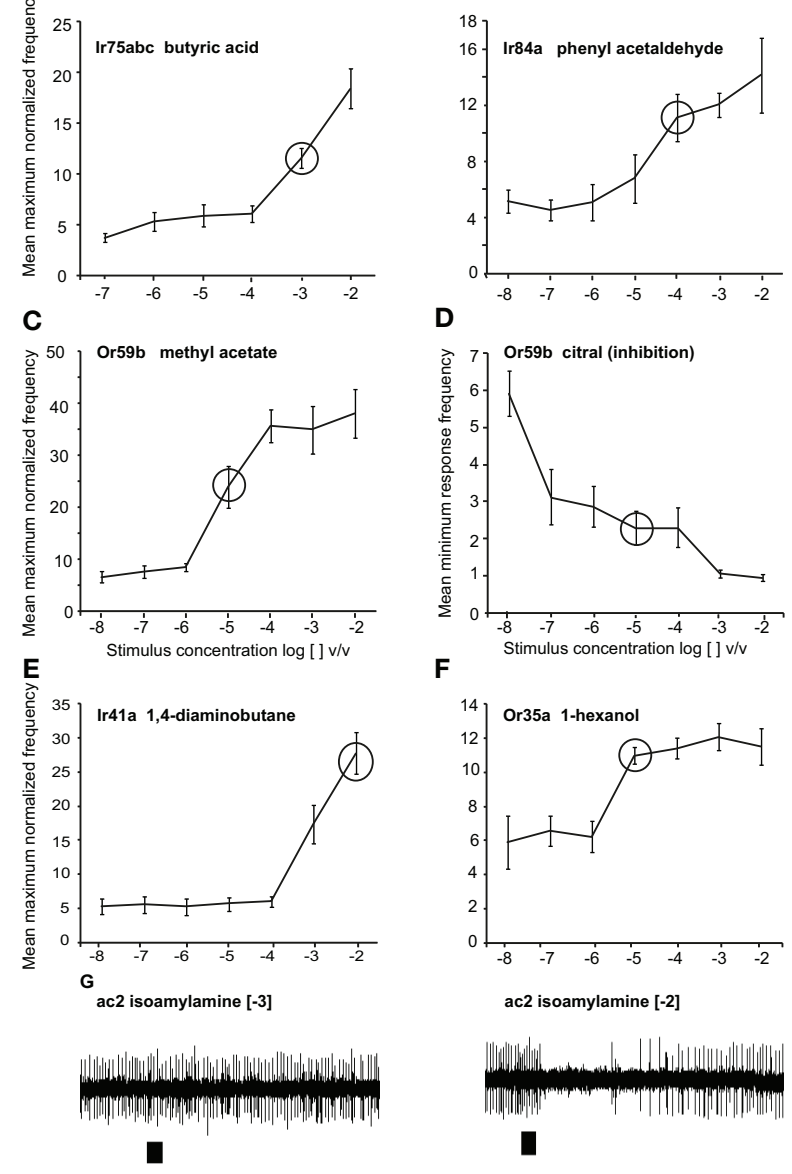

FIGURE 1 | Responses to odors at different doses. Dose-response curves presented as normalized maximum frequency response for (A) Ir75abc-expressing neurons to butyric acid $n=8-13$ (B) |r84a-expressing neurons to phenylacetaldehyde, $n=9-12$. (C), Or59b-expressing neurons to methyl acetate, $n=8-17$ (D) Or59b-expressing neurons to citral presented as the minimum frequency, $n=6-10$. (E) Ir41a-expressing neurons to 1, 4-diaminobutane $n=6-8$ (F). Or35a-expressing OSNs to 1-hexanol, $n=6-8$. (G) Representative traces showing the response of OSNs of ac2 sensilla to isoamylamine at two different concentrations (responses to lower concentrations were not observed). Please note that while only Ir41a-expressing neurons are excited by 1,4-diaminobutane in this sensillum (ac2), all neurons are inhibited by isoamylamine, and we thus label the inhibitory responses with the entire sensillum label.

to an Eppendorf tube and placed in the bottom of a PEEK vial $(4.6 \mathrm{~cm} \times 2.5 \mathrm{~cm} \times 2.5 \mathrm{~cm}$ dimensions $)$. Each vial was sealed with a stainless steel plug (Olsson et al., 2011). The pulse duration, inter-stimulus interval and number of pulses were adjusted through a custom built Labview program (Olsson et al., 2011). The odors were delivered from the headspace via Teflon tubing $150 \mathrm{~cm}$ long with an inner diameter of $1 \mathrm{~mm}$ and positioned as close as possible $(\sim 1.5 \mathrm{~cm})$ to the antennae. The flow rate of air was $0.5 \mathrm{~L} / \mathrm{min}$. For stimulation, the stimulus system was connected to the IDAC (Syntech, Ockenfels, Germany) and through USB connection to a PC. Stimulation was controlled by an OEM (EDP 0504, thinXXS) pump control system and DAQ (USB 6008 
data acquisition hardware, National Instruments, Austin, TX, USA) with custom-built Labview 8.5 software (built by Daniel Veit; National Instruments). For frequency stimulation the on time was $50 \mathrm{~ms}$ for OR- and Gr21a-OSNs and the off time was adjusted from $950 \mathrm{~ms}$ or $50 \mathrm{~ms}$ for $1-10 \mathrm{~Hz}$, respectively. For IROSNs stimulated with $[-4]$ and $[-3]$ stimulus concentrations, the pump on time was $200 \mathrm{~ms}$ and off time 800,300 , or $50 \mathrm{~ms}$ for $1-4 \mathrm{~Hz}$, respectively. At [-2], the protocol was identical to the OR-OSNs and Gr21a-OSNs. The consistency of odor delivery for different pulse durations and frequencies was confirmed using PID (200a, Aurora Scientific Ontario, Canada).

\section{DATA ANALYSIS}

All raw spike data were acquired and converted to digital spikes using Autospike 3.7 (Syntech). Co-localized neurons were identified based on spike amplitude. Peri-stimulus time histograms (PSTHs) were obtained by averaging spike activities in $25 \mathrm{~ms}$ bins from the start of the stimulation and normalized to the average frequency for $2 \mathrm{~s}$ before stimulation (Olsson et al., 2011; Sargsyan et al., 2011). The OSN responses between consecutive pulses were compared using repeated measure ANOVA by assessing the normalized mean of area under curve (AUC) spike frequency per each stimulus duration, i.e. pump on time + off time. Consecutive pulses were normalized to the response of 1st pulse. Between treatments, a Mann-Whitney $U$ test or $t$-test was used depending on the normality of the data. To evaluate the capacity of receptors to resolve pulsed stimuli, we visualized the response using normalized peri-stimulus histograms and quantified the \% return to the spontaneous activity (baseline), using the ratio between the first value in the 2 nd pulse and the maximum peak value of the first (previous) pulse converted to a percentage: Percent return to baseline $=1-(1$ st value of the 2 nd pulse/maximum frequency of the 1st pulse) $\times 100$ (Bau et al., 2002). A One-Way ANOVA followed by a Tukey post-hoc test was performed to determine if the return to baseline was significantly reduced between the different stimulation frequencies. Latency was measured as the time from the onset of the odor stimulus to the maximum response frequency (mechanical delay was not considered). Response width was calculated as the time between half-maximal response for excitation and half-minimal response in the case of inhibition. Spearman's correlation was used to assess the relationship between repeated pulses and latency as well as between response width and intensity with stimulus duration. All analyses were performed using SPSS version 17 (IBM Corporation, Armonk, New York, US).

\section{RESULTS}

\section{RESPONSE DYNAMICS OF DIFFERENT SENSORY NEURONS TO VARYING STIMULUS DURATIONS}

We first assessed the response of OSNs carrying ORs, Gr21a, or IRs to key ligands presented with varying stimulus durations at concentrations found in the linear portion of the dose-response curve for each OSN (Figure 1). OSNs expressing Or59b housed in basiconic sensillum type ab2 were stimulated with methyl acetate at $[-5]$ concentration, with stimulus durations varying from $10 \mathrm{~ms}$ to $2 \mathrm{~s}$. At $20 \mathrm{~ms}$, the mean normalized frequency of Or59b-expressing OSNs was greater than the spontaneous activity ( $t=3.482, P=0.005)$, indicating that a $20 \mathrm{~ms}$ stimulation was sufficient to elicit a response (Figure 2A asterisk right). A maximal stimulus response was obtained with a $50 \mathrm{~ms}$ stimulation $(P<0.05)$, however, stimulations of 1 s or more significantly reduced the OSN response maximum $(t=3.482, P=0.005$, mean normalized maximum frequency for $500 \mathrm{~ms}$ vs. $1 \mathrm{~s}$ stimulation and $t=5.047, P<0.001$ for $500 \mathrm{~ms}$ vs. $2 \mathrm{~s}$, Student's $t$-test). Similar response dynamics were observed in Or35a-expressing OSNs $(t=5.007, P<0.001$ mean normalized maximum frequency for $500 \mathrm{~ms}$ vs. $2 \mathrm{~s}$ stimulation; Figure 2B). Adaptation to long stimulus durations ( $>1 \mathrm{~s}$ ) was also apparent for Or22a-OSNs (data not shown). There was also a positive and significant correlation between response width at half-maximal response and stimulus duration for both OR-expressing OSNs $(r=0.853, P<$ 0.001 for Or59b-OSNs and $r=0.93, P<0.001$ for Or35a-OSNs; both Spearman's correlation Figures 2A,B left panels).

OSN expressing Ir84a (Figure 2C) were stimulated with $[-4]$ phenyl acetaldehyde and a significant response was obtained at $100 \mathrm{~ms}(P<0.001$, Mann-Whitney $U$-test; Figure 2B $)$. A maximal response was reached at $500 \mathrm{~ms}(P=0.001$, MannWhitney $U$-test, as compared to $100 \mathrm{~ms}$ ), and the maximum response intensity did not decrease at longer stimulation durations ( $t=0.605, P=0.554$ at $500 \mathrm{~ms}$ stimulation vs. $1 \mathrm{~s}$, and $t=0.394, P=0.699$ for $500 \mathrm{~ms}$ vs. $2 \mathrm{~s}$; Student's $t$-test). The response of Ir75abc-expressing neurons was similar when stimulated with [-3] butyric acid, (significant response at $100 \mathrm{~ms}$; Mann-Whitney U, $P=0.016$, Figure 2D), and reached a maximum response at $500 \mathrm{~ms}(t=2.286, P=0.036$ compared to $100 \mathrm{~ms})$. Furthermore, the response did not change at longer stimulus durations $(t=0.096, P=0.924,500 \mathrm{~ms}$ vs. $1 \mathrm{~s}, t=$ $0.068, P=0.946,500 \mathrm{~ms}$ vs. $2 \mathrm{~s}$; Figure 2D right panel). There was also a positive and significant correlation between stimulus duration and response width at half maximal response $(r=$ $0.905, P<0.001$ for Ir84a-OSNs, and $r=0.917, P<0.001$ for Ir75abc-OSNs, Spearman's correlation; Figures 2C and D left panel). Similarly, the Ir41a-OSN response to 1,4-diaminobutane at $[-2]$ did not show adaptation at longer stimulus durations $(t=0.073, P=0.944$ for $500 \mathrm{~ms}$ vs. $1 \mathrm{~s}$ stimulations; $t=0.01$, $P=0.992$ for $500 \mathrm{~ms}$ vs. $2 \mathrm{~s}$ stimulations).

OSNs expressing Gr21a, which are housed in ab1 sensilla on the Drosophila antenna, respond to pure $\mathrm{CO}_{2}$ beginning at a $20 \mathrm{~ms}$ stimulation (Mann-Whitney $U$-test, $P=0.009$ Figure 2E). Peak response was obtained at $1 \mathrm{~s}(t=4.641$, $P=0.002$, Student's $t$-test compared to $20 \mathrm{~ms}$ ), while at a $2 \mathrm{~s}$ stimulation the maximum response frequency decreased significantly ( $t=2.63, P=0.02$, Student's $t$-test, $1 \mathrm{~s}$ vs. $2 \mathrm{~s}$ ). However, the response latency also became shorter with stimulus duration, decreasing from the $20 \mathrm{~ms}$ duration (with a mean half-maximal response on set time of $400 \pm 26.35 \mathrm{~ms}$ ), to $1 \mathrm{~s}$ (with a mean half maximal response on set time $300 \pm 17.67 \mathrm{~ms}$, $t=3.028, P=0.016$, Student's $t$-test; Figure 2E left panel). This is opposite to both OR- and IR-expressing OSNs, where there was no difference (Figures 2A-D). Similarly, the response width also increased with stimulus duration $(r=0.781, P<0.001$, Spearman's correlation, Figure 2E left panel).

Increasing stimulus concentrations reduced the duration required to elicit a response regardless of the receptor expressed. 

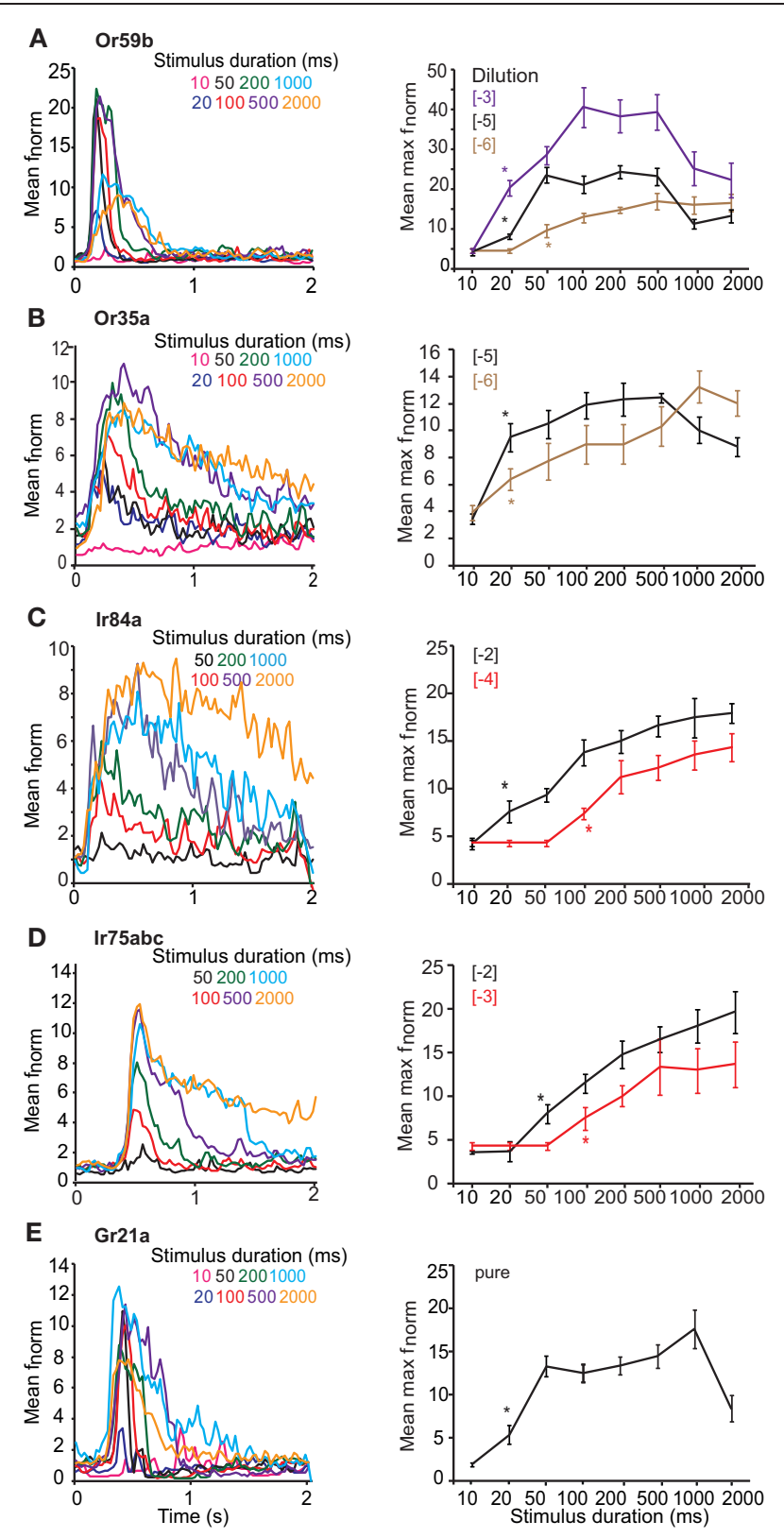

FIGURE 2 | Response of OSNs to varying stimulus durations. (A, left) Mean peri-stimulus time histograms (PSTHs, $25 \mathrm{~ms}$ bins) showing the response of Or59b-expressing OSNs to various stimulus durations of log [-5] $\mathrm{v} / \mathrm{v}$ methyl acetate. (A, right) Mean normalized maximum frequency for Or59b-expressing neurons plotted vs. stimulus duration $(n=8-15)$ for three different concentrations. Asterisks indicate the minimum stimulus duration that elicited a significant response, $P<0.05$. (B, left) Mean peri-stimulus time histograms as in (A) showing the response of Or35a-expressing OSNs to various stimulus durations of $\log [-5] \mathrm{v} / \mathrm{V}$ 1-hexanol. (B, right) Mean normalized maximum frequency for Or35a-expressing neurons plotted versus stimulus duration for log [-5] and $[-6] \mathrm{v} / \mathrm{v}$ of 1 -hexanol $(n=6-14)$. (C, left) Response of Ir84a-expressing neurons to various durations of log [-4] v/v phenyl acetaldehyde as in (A), $n=8-10$ (C, right) as in A for two different concentrations. (D, left) Response of Ir75abc-expressing neurons to various durations of $\log [-3] \mathrm{v} / \mathrm{v}$ butyric acid $(n=6-15)$ and (D, right) as in (C). (E) Response of Gr21a-expressing neurons to pure $\mathrm{CO}_{2}$ at different stimulus durations $(n=6-10)$.
For example, Or59b-OSNs required $50 \mathrm{~ms}$ at $[-6]$ to elicit a significant response $(t=2.486, P=0.025$; Figure 1A right), but only $20 \mathrm{~ms}$ at $[-3](P<0.001$, Mann-Whitney $U$-test, asterisk in Figure 2A right). Similarly, Ir84a-expressing OSNs stimulated with phenyl acetaldehyde at [-2] required only $20 \mathrm{~ms}$ to elicit a significant response (Mann-Whitney $\mathrm{U}, P=0.02$, Figure 2C), while Ir75abc-expressing OSNs required a $50 \mathrm{~ms}$ stimulation when the concentration of butyric acid increased by $10 \times[-2]$ (Mann-Whitney $\mathrm{U}, P=0.002$, Figure 2D, asterisk right).

However, the dose-dependency of OSN adaptation to long stimulus durations was dependent on the receptor expressed. At $[-6]$ long stimulus durations did not reduce the response of Or59b-expressing OSNs $(t=0.292, P=0.776$ for $500 \mathrm{~ms}$ vs. $1 \mathrm{~s} ; t=0.33, P=0.745$ for $500 \mathrm{~ms}$ vs. $2 \mathrm{~s}$ ) or Or35a-expressing OSNs $(t=1.151, P=0.147$ for $500 \mathrm{~ms}$ vs. $1 \mathrm{~s} ; t=0.948, P=$ 0.356 for $500 \mathrm{~ms}$ vs. $2 \mathrm{~s}$; Figures 2A,B right). However, at [-3] concentration, stimulations of $1 \mathrm{~s}$ or more significantly reduced the Or59b-expressing OSN response maximum $(t=2.235, P=$ 0.045 for $500 \mathrm{~ms}$ vs. $1 \mathrm{~s} ; t=2.658, P=0.021$ for $500 \mathrm{~ms}$ vs. $2 \mathrm{~s}$, Figure 2A). In contrast, longer stimulus durations did not reduce the response of IR-expressing OSNs regardless of concentration (Ir84a-expressing OSNs at [-2]: Mann-Whitney $\mathrm{U}, P=0.847$ for $500 \mathrm{~ms}$ vs. $1 \mathrm{~s}$; Ir75abc-expressing OSNs at $[-2]: t=0.644$, $P=0.531$ for $500 \mathrm{~ms}$ vs. $1 \mathrm{~s}$; Ir4la-expressing OSNs at $[-2], t=$ $0.073, P=0.944$ for $500 \mathrm{~ms}$ vs. $1 \mathrm{~s}$; Figures 2C,D right panels).

\section{PULSE RESOLUTION OF DIFFERENT SENSORY NEURONS}

After investigating the response of OSNs to various stimulus durations, we presented the neurons with repeated stimulations of varying frequency. The latency to repeated stimulations at $1 \mathrm{~Hz}$ increased for all OSN types $(r=0.742, P<0.001$ for Or59b-OSNs; $r=0.94, P<0.001$ for Gr21a-OSNs; $r=0.787$, $P<0.001$ for Ir75abc-OSNs; $r=0.652, P<0.001$ for Ir84aOSNs; Spearman's correlation; Figures 3A-E). However, a variability in latency was observed between the tested OSNs; e.g., Ir75abc-OSNs showed more delayed time to maximum than all other neurons tested, $P<0.001$, ANOVA followed by Tukey post-hoc test (Figure 3E). At $100 \times$ stimulus concentrations or a $5 \mathrm{~s}$ interstimilus interval, the latency for Or59b-expressing OSNs did not change with repeated stimulation $(r=0.09, P=0.475$; $r=-0.006, P=0.952$, respectively, Spearmans's correlation; Figure 3F). Similarly, Ir75abc-expressing OSN response onset recovered with a higher concentration $(r=0.01, P=0.90$, Spearmans's correlation). However, at $5 \mathrm{~s}$ interstimulus intervals the response onset became significantly faster for the later pulses $(r=-0.885, P<0.001$, Spearmans's correlation; Figure 3G).

The latency also decreased with subsequent stimulations of $\mathrm{CO}_{2}$ for Gr21a-expressing OSNs at $5 \mathrm{~s}$ interstimulus intervals $(r=-0.976, P<0.001$, Spearmans's correlation; Figure 3H). In summary, this shows that changes in response onset kinetics to repeated stimuli are similar across all tested OSNs and response latencies can be regulated either by altering stimulus concentrations or inter-stimulus intrervals.

Ir84a and Ir75abc-OSNs, housed in ac4 and ac3 sensilla respectively, could resolve repeated $200 \mathrm{~ms}$ pulses of [-4] and [-3] stimulus concentrations, respectively, up to $4 \mathrm{~Hz}$ (the maximum testable frequency due to stimulation length). The mean 

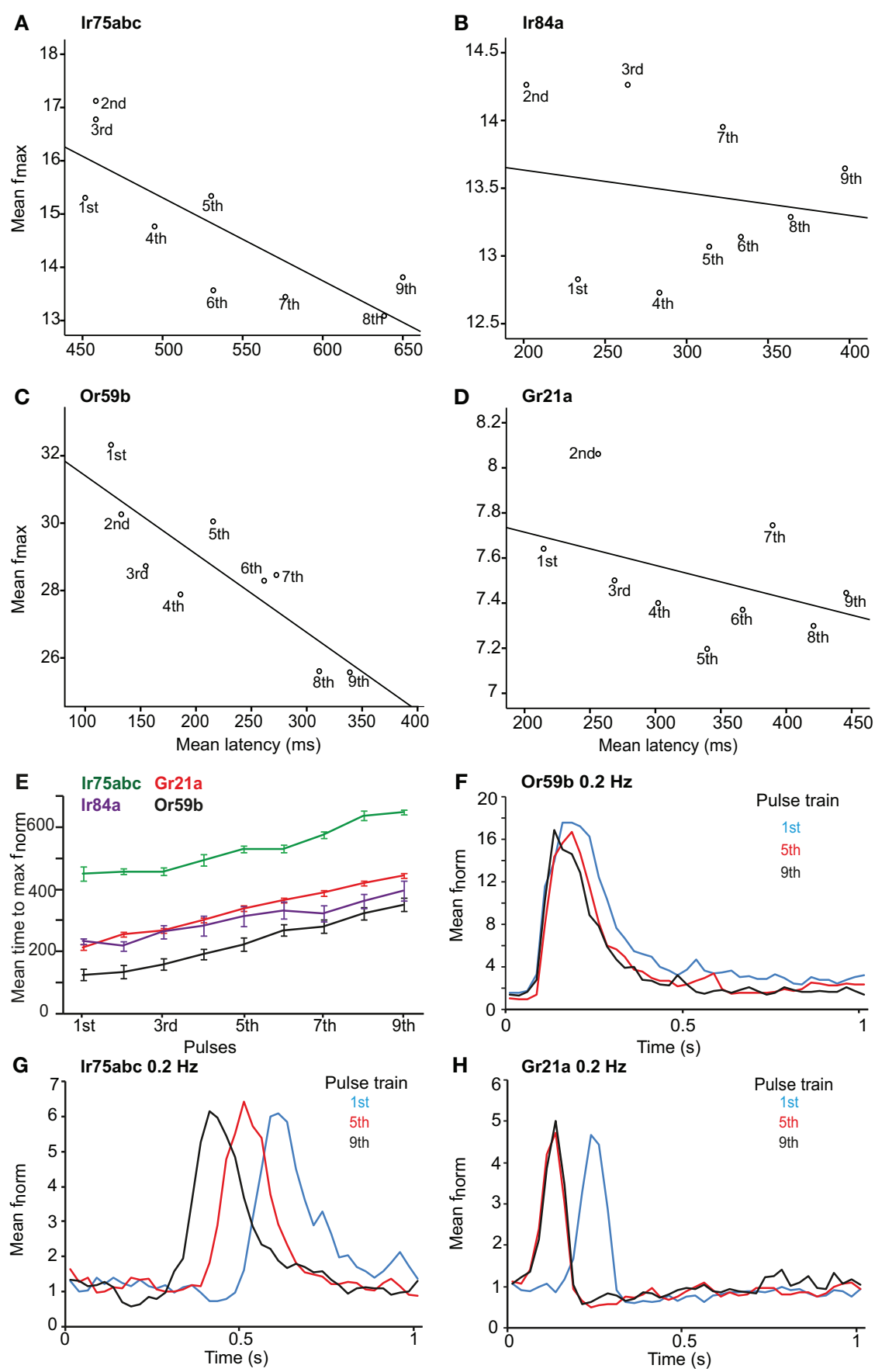

FIGURE 3 | Latency and maximum response of OSNs to repeated stimuli. Maximum response frequency vs. time to peak (latency), with best fit line, for OSNs carrying various receptors in response to repeated $1 \mathrm{~Hz}$ stimulations. (A) Ir75abc, (B) Ir84a, (C) Or59b and (D) Gr21a. Pulse number (1-9) indicated below each point. (E) Mean time

return to base line during repeated stimulation was significantly reduced at $4 \mathrm{~Hz}$ as compared to 1 and $2 \mathrm{~Hz}$ stimulation, $(P<0.05$ ANOVA followed by Tukey post-hoc test; Figures 4 A,B). At an increased concentration of $[-2]$, Ir75abc OSNs could resolve pulsed stimuli up to $5 \mathrm{~Hz}$ at a $50 \mathrm{~ms}$ pulse duration (Figure 4C).
Gr21a-expressing OSNs housed in ab1 sensilla resolved intermittent pulses of $\mathrm{CO}_{2}$ as fast as $8 \mathrm{~Hz}$ with no significant difference in return to baseline between $1 \mathrm{~Hz}$ and $5 \mathrm{~Hz}$ stimulations. At $8 \mathrm{~Hz}$, the mean return to base line was significantly reduced, and at $10 \mathrm{~Hz}$ only $2.4 \%$ recovery to the base line occurred 

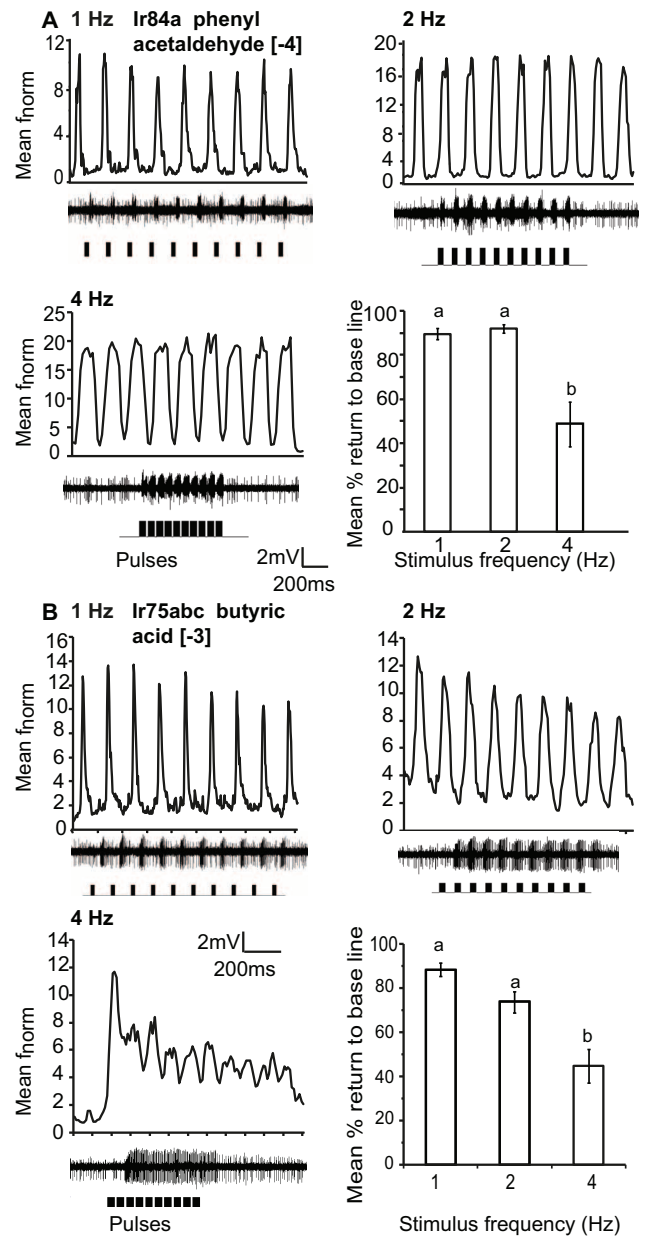

C $1 \mathrm{~Hz}$ Ir75abc butyric

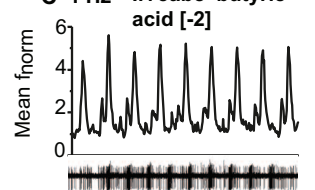

$2 \mathrm{~Hz}$

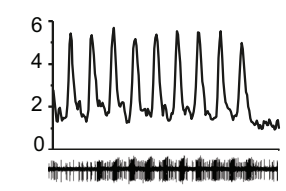

1111111111
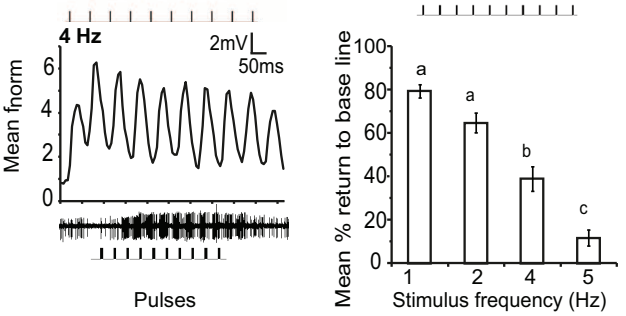

FIGURE 4 | Response of OSNs to repeated stimulus pulses at varying frequencies. (A) Average normalized PSTH responses for Ir84a-expressing neurons in response to repeated pulses of log $[-4] \mathrm{v} / \mathrm{v}$ phenyl acetaldehyde at listed frequencies. Traces below each panel show sample $200 \mathrm{~ms}$ recordings. Square pulses indicate stimulus presentation. The final panel shows the mean percent return to base line across all pulses at listed frequencies; error bars indicate SEM (ANOVA, $P<0.05$, followed by Tukey post-hoc, $n=7-9)$. (B) Response of Ir75abc-expressing neurons to repeated stimulations of $\log [-3] \mathrm{v} / \mathrm{v}$ butyric acid stimulation as in (A) (ANOVA, $P<0.05$, followed by Tukey post-hoc $(n=8-10)$. (C) Response as in (B) to a $10 \times$ concentration of butyric acid (log [-2]); ANOVA, $P<0.05$, followed by Tukey post-hoc, $n=14-15)$.
$(P<0.001$, ANOVA followed by Tukey post-hoc test; Figures 5A and B). Gr21a-expressing OSNs also exhibited short term adaptation based on AUC (see "Materials and methods") that was frequency dependent, i.e. at $1 \mathrm{~Hz}$ stimulation the 9th pulse resulted in a significantly reduced response compared to the 1st pulse (repeated measure ANOVA, $P<0.001$ ), while at $2 \mathrm{~Hz}$ the 4 th pulse was reduced $(P=0.039)$, at $4 \mathrm{~Hz}$ the 5 th $(P=0.001)$, and at 5 and $8 \mathrm{~Hz}$ the 2nd $(P<0.01$, repeated measure ANOVA; Figure 5 asterisks).

\section{PULSE RESOLUTION OF STIMULI ELICITING OPPOSITE RESPONSE POLARITY}

We also tested the pulse following capacity to single excitatory and inhibitory odor ligands in Or59b-expressing OSNs. We applied [-5] methyl acetate as an excitatory and [-5] citral as an inhibitory ligand. Or59b-expressing OSNs could resolve the excitatory stimulus up to $5 \mathrm{~Hz}$ (Figure 6A). The mean return to base line was significantly reduced at $5 \mathrm{~Hz}$ stimulation as compared to 1 and $2 \mathrm{~Hz}(P<0.05$, ANOVA followed by Tukey post-hoc test; Figure 6C). However, the pulse resolution was also affected by concentration, as a $100 \times$ increase in concentration reduced the pulse resolution to $2 \mathrm{~Hz}(P<0.05)$. In contrast to the excitatory responses, Or59b-cells were able to resolve pulses of the inhibitory ligand citral only up to $2 \mathrm{~Hz}$, and at 4 and $5 \mathrm{~Hz}$ the OSNs showed total inhibition and did not recover when stimulated repeatedly with the inhibitory ligand $(P<0.05$ ANOVA followed by
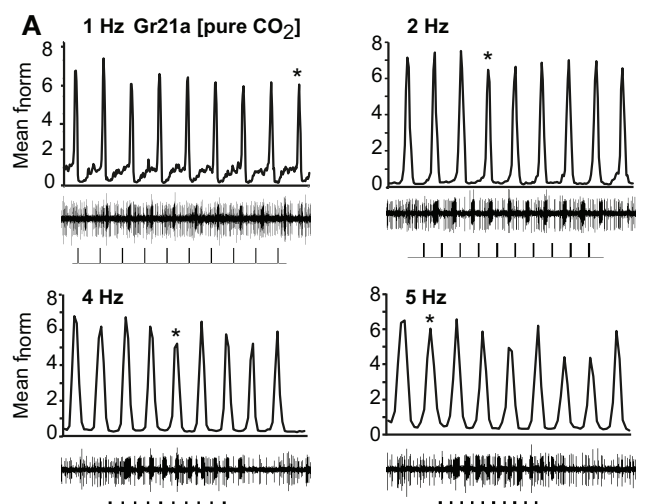

11111111111

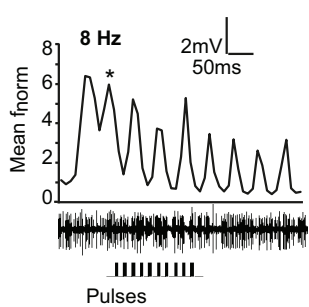

IIIIIIIII

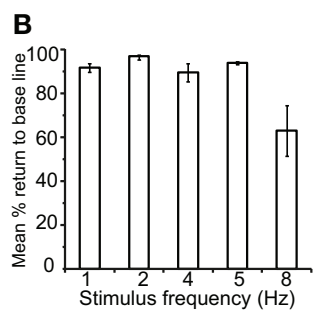

FIGURE 5 | Response of Gr21a-expressing OSNs to repeated stimulus pulses at varying frequencies. (A) Average normalized PSTH responses of $\mathrm{Gr} 21 \mathrm{a}$-expressing neurons to repeated pulses of pure $\mathrm{CO}_{2}$ at listed frequencies. Traces below each panel show sample $50 \mathrm{~ms}$ recordings. Square pulses indicate stimulus presentation. (B) Mean percent return to base line across all pulses of listed frequencies, error bars indicate SEM (ANOVA, $P<0.05$, followed by Tukey post-hoc test, $n=11-12$ ). 


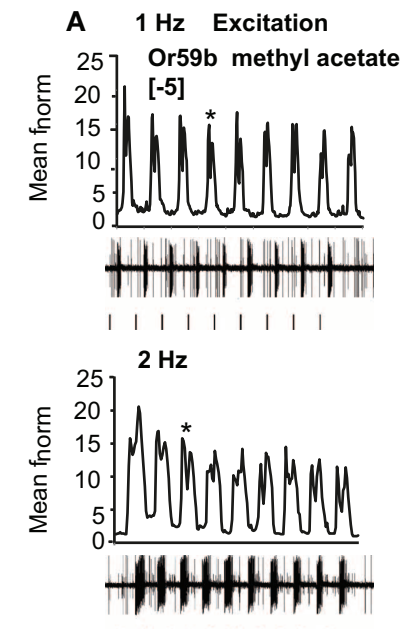

||||||||||||$|l|$
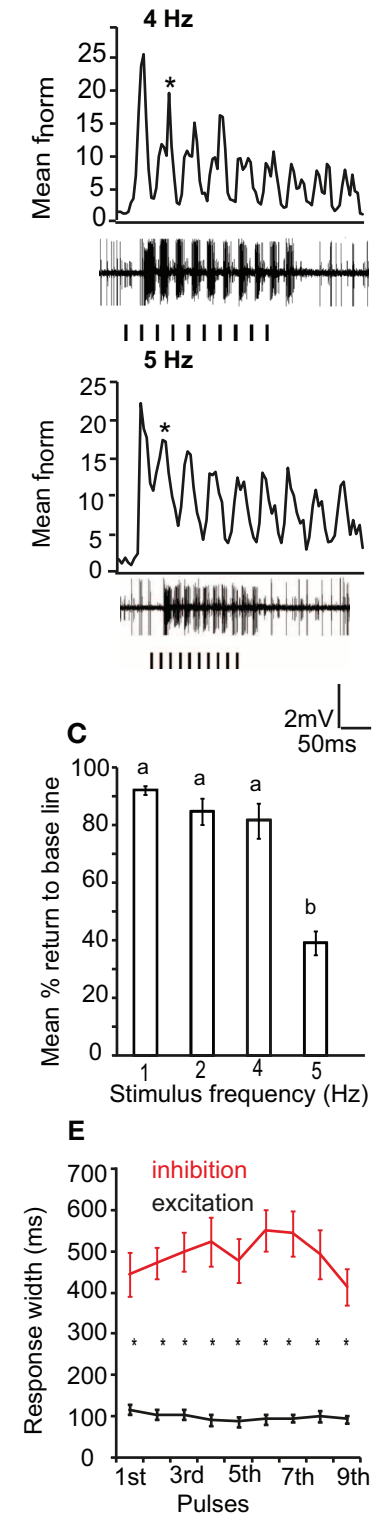
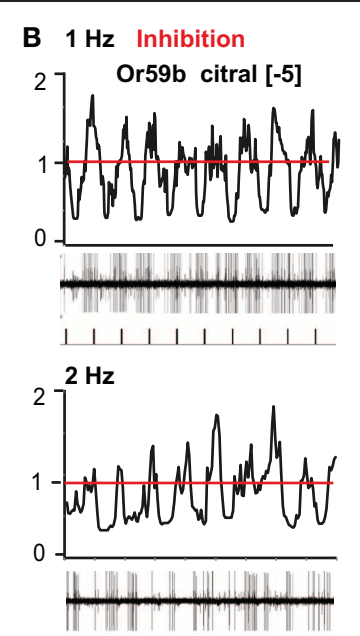

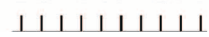

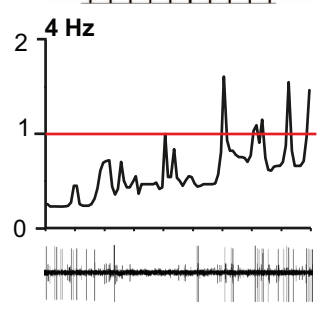

1111111111

$5 \mathrm{~Hz}$

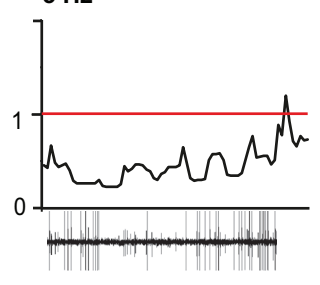

111111111
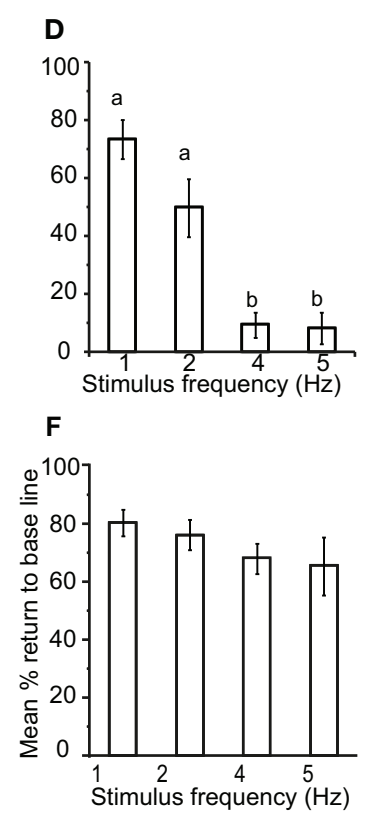

FIGURE 6 | OR-expressing OSN response polarity and pulse resolution. (A) Mean normalized PSTH response of Or59b-expressing OSNs to repeated pulses of log [-5] v/v methyl acetate at listed frequencies. Traces below each panel show sample $50 \mathrm{~ms}$ recordings. Square pulses indicate stimulus presentation. (B) Mean normalized PSTH response of

Or59b-expressing OSNs to repeated pulses of log [-5] v/v citral (an inhibitory odor) at listed frequencies as in (A). Red line indicates baseline frequency. (C) Mean percent return to base line across all pulses for Or59b-OSN response to methyl acetate, error bars indicate SEM (ANOVA, $P<0.05$, followed by Tukey post-hoc test, $n=9-13)$ and (D) as in (C) for citral (ANOVA, $P<0.05$, followed by Tukey post-hoc test, $n=13-15)$. (E) Mean response width of Or59b-expressing OSNs for excitation and inhibition. (F) Mean percent return to base line in response to a pulsed binary mixture of methyl acetate and citral, error bars indicate SEM, $(P>0.05$ ANOVA, $n=8-9)$.

Tukey post-hoc test; Figures 6B and D). The inhibitory ligand also resulted in a larger response width as compared to the excitatory ligand, even though both ligand concentrations were at similar points in the dose response curve (see Figure 1). This indicates that a given OSN response to an inhibitory or excitatory ligand can differ not only in polarity but also in temporal dynamics (Figure 6E). Furthermore, Or59b-OSNs showed short-term adaptation to the excitatory ligand that was frequency dependent (repeated measure ANOVA, $P<0.05$ ). At increasing frequencies, short-term adaptation occurred earlier in the stimulus train (Figure 6A asterisk). In contrast, we did not find short-term adaptation based on response width to the inhibitory ligand (repeated measure ANOVA, $P>0.05$ ).

We also asked if the total inhibition of the neuron at high frequencies of citral $(>4 \mathrm{~Hz})$ could interfere with odor coding of the excitatory ligand when presented simultaneously to the OSN. We thus stimulated the neurons with the binary mixture of the two ligands at the concentrations listed above. Stimulation with the two component blend resulted in an improved pulse resolution over either separate odor, with no significant difference in pulse resolution between 1 and $5 \mathrm{~Hz}(P>0.05$ ANOVA (Figure 6F). The effect of response polarity on pulse resolution was also observed in OSNs that express IRs. Ir41a-OSNs exhibited an excitatory response to $50 \mathrm{~ms}$ pulses of [-2] 1,4-diaminobutane and resolved pulsed stimuli as fast as $2 \mathrm{~Hz}$, (ANOVA, $P<$ 0.05; Figures 7A and $\mathbf{D}$ ). However, the pulse resolution to the inhibitory ligand isoamylamine at $[-2]$ (the concentration at which the neurons are inhibited by the ligand, Figure 1G) was only maintained at $1 \mathrm{~Hz}$ (ANOVA $P<0.05$; Figures $7 \mathbf{B}$ and $\mathbf{E}$ ). In addition, the binary mixture of 1, 4-diaminobutane and isoamylamine at the same concentration $[-2]$, sharpened the response of Ir41a-OSNs especially at $4 \mathrm{~Hz}$ (Figures 7C and F).

\section{DISCUSSION}

Odor stimuli contain three elements of information: odor identity; odor intensity, and a temporal component (Hallem et al., 2004). To respond to these stimuli, insect OSNs express a wide variety of receptors. Here we investigate the response dynamics of OSNs expressing receptors from different protein families to stimuli of both different durations and frequencies. We find that ORs, IRs, and Gr21a exhibit distinct response characteristics that could increase the response range of the insect to the temporally dynamic natural odor environment.

FIGURE 6 | Continued 

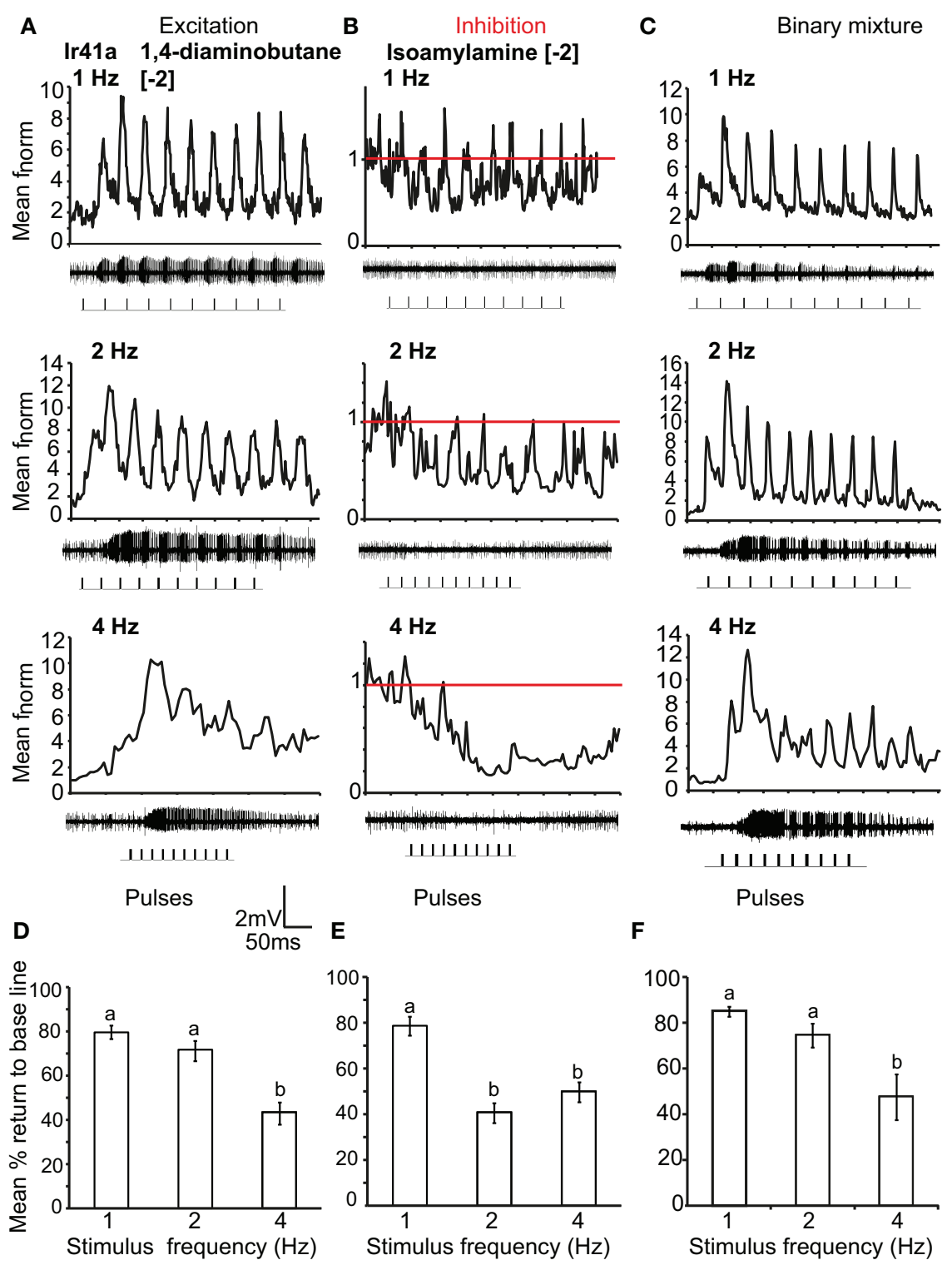

FIGURE 7 | IR-expressing OSN response polarity and pulse resolution. (A) Mean normalized PSTH response of Ir41a-expressing OSNs to repeated pulses of $\log [-2] \mathrm{V} / \mathrm{V} 1$, 4-diaminobutane at listed frequencies. Traces below each panel show sample $50 \mathrm{~ms}$ recordings. Square pulses indicate stimulus presentation. (B) As in (A) for log [-2] v/v of the inhibitory odor isoamyl amine. Red line indicates baseline frequency. (C) Mean normalized PSTH response of Ir41a-expressing OSNs to a binary mixture of 1, 4-diaminobutane and isoamyl amine at [-2] v/v. (D) Mean percent return to base line to the excitatory ligand across all pulses at listed frequencies, error bars indicate SEM (ANOVA, $P<0.05$, followed by Tukey post-hoc test, $n=7-8$ ) (E), as in (D) for log [-2] v/v of the inhibitory odor isoamyl amine (ANOVA, $P<0.05$, followed by Tukey post-hoc test; $n=7-9$ ). (F) as in (D) for the binary mixture (ANOVA, $P<0.05$, followed by Tukey post-hoc test, $n=7-9$ ).
RESPONSE DYNAMICS TO DIFFERENT STIMULUS DURATIONS ARE A FUNCTION OF RECEPTOR TYPE

We found that the response of Drosophila OSNs to varying stimulus durations (Figure 2) depends on the type of receptor expressed in that neuron. OR-expressing OSNs showed adaptation to higher concentrations of long stimulus pulses $(>1 \mathrm{~s})$, both in maximum frequency and latency. This response feature was also independent of ligand (data not shown). In contrast, when IR-expressing OSNs were tested with the same protocol, they required longer stimulus durations to respond, and there was no desensitization even up to $2 \mathrm{~s}$ stimulation either in response intensity or latency regardless of stimulus concentration. As a consequence, OSNs that express IRs are able to transmit information concerning the presence of longlasting odors in their environment better than OR-expressing OSNs. However, this could also present a trade off, because the signal transduction in these OSNs appears to be slower, as seen in Figure 3E, where the time to maximum frequency was 
longer in IR-expressing OSNs as compared to OR-expressing OSNs.

The difference in response between IR- and OR-expressing OSNs to longer pulses was not a function of stimulus presentation, which was assessed by PID (see "Materials and methods"). It is therefore a property of the OSNs themselves. Are these differences a function of the peri-receptor environment, or rather a property of internal OSN kinetics? To test this, we assessed the response of Or35a-OSNs, which are housed in coeloconic sensillum ac3 together with Ir75abc-OSNs. As with other ORexpressing OSNs, Or35a-OSNs also responded to stimulations as brief as $20 \mathrm{~ms}$ and showed desensitization at longer pulses (2s) in maximum response frequency (Figure 2B). The response kinetics of these OSNs is therefore less influenced by the environment where they are expressed and rather by intrinsic properties of the neurons themselves.

The broad protostome conservation of IRs contrasts sharply with the restriction of ORs to insect genomes. This phylogenetic evidence suggests that IRs were the first olfactory receptor repertoire in insects (Robertson et al., 2003; Croset et al., 2010). IRs are also restricted to coeloconic sensilla, whereas ORs are found in several morphological sensillum types (Gupta and Rodrigues, 1997; Goulding et al., 2000; zur Lage et al., 2003; Benton et al., 2009). Our results show that IR-expressing OSNs required longer stimulation times to respond to key odorants, and responded with lower response intensities. This could imply that IRs are less efficient and less sensitive in detecting and transducing a chemical signal. OR activation results in both ionotropic and metabotropic signaling (Wicher et al., 2008; Deng et al., 2011), while IRs are thought to be purely ionotropic (Benton et al., 2009). Iontropic signaling is also known to be less sensitive (Sato et al., 2008, 2011; Wicher et al., 2008). The requirement for higher concentrations in IR-expressing OSNs has been also shown in Yao et al. (2005). The signal transduction in Gr21a has been shown to involve $\mathrm{G} \alpha_{\mathrm{q}}$ protein, but not $\mathrm{G} \alpha_{\mathrm{s}}$ (Yao and Carlson, 2010; Deng et al., 2011). Thus, it is possible that the transduction cascade itself leads to these differences in response to varying stimulus durations.

The desensitization/adaptation at longer stimulus durations could affect the temporal accuracy of OR-expressing OSNs in reporting long-lasting odor strands, but it may also enrich the coding possibilities for odor discrimination (DeBruyne and Baker, 2008; Nagel and Wilson, 2011) by allowing the neuron to return to its resting state more quickly. This could provide additional possibilities for odor discrimination such as under background odor, or for resolution of intermittent pulsed stimuli. Adaptation extends the operating range of sensory systems, in some cases over an enormous span of stimulus intensities (Torre et al., 1995). It may also play a role in complex functions of neuronal systems such as stimulus location (Kaissling et al., 1987). Similar results were reported in the locust where the electrophysiological response of projection neurons also depended on stimulus duration (Brown et al., 2005; Mazor and Laurent, 2005). In contrast, the long-lasting response of IR-expressing OSNs could allow for close range detection while on or very near the stimulus source where stimulus durations could persist for much longer periods of time
(Murlis et al., 2000; Louis et al., 2008; Gomez-Marin et al., 2011).

\section{PULSE RESOLUTION IS RECEPTOR TYPE DEPENDENT}

The different classes of OSNs also showed differences in their pulse resolution to repeated stimuli. Brief intermittent stimuli were not detected by IR-expressing OSNs, in contrast to those expressing ORs (which could respond up to $5 \mathrm{~Hz}$ ). This response characteristic was mainly due to a difference in sensitivity, as increasing the stimulus concentration for IR-expressing OSNs improved the detection and resolution to $5 \mathrm{~Hz}$. In contrast, a $100 \times$ increase in concentration actually reduced the OR-OSNs pulse resolution. The accuracy of encoding rapidly fluctuating intermittent odorant stimuli above $5 \mathrm{~Hz}$ was significantly reduced for all OSNs regardless of receptor type. Similarly, other insects resolved up to $5 \mathrm{~Hz}$ pulses of general odors or pheromones (e.g., Lemon and Getz, 1997; Barrozo and Kaissling, 2002; Bau et al., 2002), even at the antennal lobe (e.g., Christensen and Hildebrand, 1997; Lei and Hansson, 1999; Lei et al., 2009).

Short term adaptation and latency to peak response to repeated stimuli were independent of the receptor expressed in the OSN (Figures 3A-E). In addition, the time to peak response and the response intensity were recovered in all OSNs either by increasing the inter-stimulus interval to $5 \mathrm{~s}$ or by increasing the concentration. This suggests that adaptation to repeated stimulation is a general feature of all OSNs, regardless of the receptor expressed. Adaptation is assumed to be an early step in information processing and decision making (Kaissling et al., 1987; Baker et al., 1988; Dolzer et al., 2003; Theodoni et al., 2011), and appears to affect the response of all OSN types in a similar manner.

\section{RESPONSE POLARITY AFFECTS PULSE RESOLUTION}

Both OR- and IR-expressing OSNs were unable to resolve pulsed inhibitory ligands at frequencies as high as excitatory ligands (Figures 6B and $\mathbf{7 B}$ ). This could be because the response inhibition lasted longer than excitation (Figure 6E), even though the concentrations tested were at the same point in the dose response curve (Figure 1). According to Ghatpande and Reisert (2011), fast response termination improves pulse resolution. Similarly, Su et al. (2011) showed that the inhibitory responses of OSNs lasted much longer than their excitatory responses, but the reason for this difference is not clear. Interestingly, a mixture of both excitatory and inhibitory odors improved pulse resolution at high frequencies (Figures 6F and $7 \mathbf{F}$ ). As a consequence, OSNs may respond to intermittent blends at faster rates, which may increase their ability to track complex natural stimuli.

The fast-terminating biphasic response exhibited by Gr21a-OSNs in response to $\mathrm{CO}_{2}$ stimulation could be the reason why Gr21a-OSNs resolved more rapid stimulations as compared to OR- and IR-expressing OSNs (Figure 5). A biphasic response improved pulse resolution in antennal lobe neurons (Lei and Hansson, 1999). Besides the OSN itself, the chemistry of $\mathrm{CO}_{2}$ could also contribute to 
better pulse resolution as it will readily hydrate to bicarbonate (Kwon et al., 2007), and the degree of odor clearing is one of the challenges for resolving rapidly fluctuating odorant stimuli (Ishida and Leal, 2005; Ghatpande and Reisert, 2011).

\section{CONCLUSION}

Terrestrial olfaction requires the tracking of brief, intermittent airborne stimuli in a turbulent and dynamic environment. Fast reaction times to pockets of clean air are suggested to be behaviorally important for successful and rapid source location; hence, the selection over evolutionary time for sensitive and high-fidelity odor strand detection and resolution in the insect olfactory system is crucial (Baker and Vickers, 1997). Equally, the temporal structure of olfactory information has been shown to be critical for odor coding in a variety of systems (Laurent et al., 2001). Here we show that IR-expressing OSNs are better in detecting longlasting odor pulses, but they are less sensitive. That could suggest that they are better at close range odor detection where odor-OR interaction time is not a limiting factor (high molecular flux). In contrast OR-expressing neurons are more sensitive and better at resolving brief (low molecular flux) pulsed stimuli. This diversity in temporal characteristics could provide a broad palette of response kinetics for the insect olfactory system to respond to

\section{REFERENCES}

Ai, M., Min, S., Grosjean, Y., Leblanc, C., Bell, R., Benton, R., et al. (2010). Acid sensing by the Drosophila olfactory system. Nature 468, 691-695.

Baker, T. C., Hansson, B. S., Löfsted, C., and Löfqvist, J. (1988). Adaptation of antennal neurons in moths is associated with cessation of pheromone-mediated upwind flight. Proc. Natl. Acad. Sci. U.S.A. 85, 9826-9830.

Baker, T. C., and Vickers, N. J. (1997). "Pheromone-mediated flight in moths," In Pheromone Research: New Directions. ed R. T. Cardé (Minks AK. New York: Chapman and Hall), 248-264.

Barrozo, R. B., and Kaissling, K.-E. (2002). Repetitive stimulation of olfactory receptor cells in female silkmoths Bombyx mori L. J. Insect Physiol. 48, 825-834.

Bau, J., Justus, K. A., and Carde', R. T. (2002). Antennal resolution of pulsed pheromone plumes in three moth species. J. Insect Physiol. 48, 433-442.

Benton, R., Sachse, S., Michnick, S. W., and Vosshall, L. B. (2006). Atypical membrane topology and heteromeric function of Drosophila odorant receptors in vivo. PLoS Biol. 4:e20. doi: 10.1371/journal.pbio.0040020

Benton, R., Vannice, K. S., GomezDiaz, C., and Vosshall, L. B. (2009). Variant ionotropic glutamate receptors as chemosensory receptors in Drosophila. Cell 136, 149-162.

Brown, S. L., Joseph, J., and Stopfer, M. (2005). Encoding a temporally structured stimulus with a temporally structured neural representation. Nat. Neurosci. 8, 1568-1576.

Christensen, T. A., and Hildebrand, J. G. (1997). Coincident stimulation with pheromone components improves temporal pattern resolution in central olfactory neurons. J. Neurophysiol. 77, 775-781.

Clyne, P. J., Warr, C. G., Freeman, M. R., Lessing, D., Kim, J., and Carlson, J. R. (1999). A novel family of divergent seven-trans- membrane proteins: candidate odorant receptors in Drosophila. Neuron 22, 327-338.

Couto, A., Alenius, M., and Dickson, B. J. (2005). Molecular anatomical and functional organization of the Drosophila olfactory system. Curr. Biol. 15, 1535-1547.

Croset, V., Rytz, R., Cummins, S. F., Budd, A., Brawand, D., Kaessmann, H., et al. (2010). Ancient protostome origin of chemosensory ionotropic glutamate receptors and the evolution of insect taste and olfaction. PLoS Genet. 6:e1001064. doi: 10.1371/journal.pgen.1001064

DeBruyne, M., and Baker, T. C. (2008). Odor detection in insects: volatile codes. J. Chem. Ecol. 34, 882-897.

Deng, Y., Zhang, W., Farhat, K., Oberland, S., Gisselmann, G., and Neuhaus, E. M. (2011). The

the high-dimensional temporal input found in an insect's odor environment.

IRs are the only receptors found in basal insects and conserved between unicellular and multicellular organisms (Croset et al., 2010). ORs appear to have derived from the gustatory receptor family (Robertson et al., 2003; Nordström et al., 2011), which is present in insects as well as in aquatic arthropods such as water fleas (Peñalva-Arana et al., 2009). Besides increasing the diversity of chemicals that could be detected, OR-OSNs also allow the olfactory system to rapidly detect and transduce brief airborne odor information. This is especially important for flying insects, for which stimulus contact is brief and fast response in time is most critical. OR-expressing OSNs were indeed more sensitive to intermittent stimuli than IRs and Gr21a. The sensitive and fast neuronal response observed in OR-expressing OSNs could result from Orco-dependent transduction, which may have evolved through selective pressure to increase sensitivity and speed of odor detection while in flight.

\section{ACKNOWLEDGMENTS}

This study was supported by the International Max Planck Research School (Merid N. Getahun) and the Max Planck Society (Dieter Wicher, Bill S. Hansson, and Shannon B. Olsson).

stimulatory Gas protein is involved in olfactory signal transduction in Drosophila. PLoS ONE 6:e18605. doi: 10.1371/journal.pone.0018605

Dolzer, J., Fischer, K., and Stengl, M. (2003). Adaptation in pheromone sensitive trichoid sensilla of the hawkmoth Manduca sexta. J. Exp. Biol. 206, 1575-1588.

Ghatpande, A. S., and Reisert, J. (2011). Olfactory receptor neuron responses coding for rapid odour sampling. J. Physiol. 589, 2261-2273.

Gomez-Marin, A., Greg, J., Stephens, G. J., and Louis, M. (2011). Active sampling and decision making in Drosophila chemotaxis. Nat. Commun. 2, 441.

Goulding, S. E., zur Lage, P., and Jarman, A. P. (2000). Amos, a proneural gene for Drosophila olfactory sense organs that is regulated by lozenge. Neuron 25, 69-78.

Gupta, B. P., and Rodrigues, V. (1997). Atonal is a proneural gene for a subset of olfactory sense organs in Drosophila. Genes Cells 2, 225-233.

Hallem, E. A., Ho, M. G., and Carlson, J. R. (2004). The molecular basis of odor coding in the Drosophila antenna. Cell 117, 965-979.

Ishida, Y., and Leal, W. S. (2005). Rapid inactivation of a moth pheromone. Proc. Natl. Acad. Sci. U.S.A. 102, 14075-14079.

Jones, W. D., Cayirlioglu, P., Kadow, I. G., and Vosshall, L. B. (2007). Two chemosensory receptors together mediate carbon dioxide detection in Drosophila. Nature 445, 86-90.

Kaissling, K. E., Zack Strausfeld, C., and Rumbo, E. R. (1987). Adaptation processes in insect olfactory receptors. Mechanisms and behavioral significance. Ann. N.Y. Acad. Sci. 510, 104-112.

Kwon, J. Y., Dahanukar, A., Weiss, L. A., and Carlson, J. R. (2007). The molecular basis of $\mathrm{CO}_{2}$ reception in Drosophila. Proc. Natl. Acad. Sci. U.S.A. 104, 3574-3578.

Laurent, G., Stopfer, M., Friedrich, R. W., Rabinovich, M. I., Volkovskii, A., and Abarbanel, H. D. (2001). Odor encoding as an active, dynamical process: experiments, computation, and theory. Annu. Rev. Neurosci. 24, 263-297.

Lei, H., and Hansson, B. S. (1999). Central processing of pulsed pheromone signals by antennal lobe neurons in the male moth Agrotis segetum. J. Neurophysiol. 81, 1113-1122.

Lei, H., Riffell, J. A., Gage, S. L., and Hildebrand, J. G. (2009). Contrast enhancement of stimulus intermittency in a primary olfactory network and its behavioral significance. J. Biol. 8, 21.

Lemon, W. C., and Getz, W. M. (1997). Temporal resolution of general odor pulses by olfactory sensory neurons in American cockroaches. J. Exp. Biol. 200, 1809-1819.

Louis, M., Huber, T., Benton, R., Sakmar, T. P., and Vosshall, L. B. 
(2008). Bilateral olfactory sensory input enhances chemotaxis behavior. Nat. Neurosci. 11, 187-199.

Mazor, O., and Laurent, G. (2005). Transient dynamics versus fixed points in odor representations by locust antennal lobe projection neurons. Neuron 48, 661-673.

Murlis, J., Willis, M. A., and Carde', R. T. (2000). Spatial and temporal structures of pheromone plumes in fields and forests. Physiol. Entomol. $25,211-222$.

Nagel, K. I., and Wilson, R. I. (2011). Biophysical mechanisms underlying olfactory receptor neuron dynamics. Nat. Neurosci. 14, 208-216.

Nordström, K. J., Almen, M. S., Edstam, M. M., Fredriksson, R., and Schioth, H. B. (2011). Independent HHsearch, Needleman-Wunschbased and motif analyses reveals the overall hierarchy for most of the G protein-coupled receptor families. Mol. Biol. Evol. 28, 2471-2480.

Olsson, S. B., Getahun, M. N., Wicher, D., and Hansson, B. S. (2011). Piezo-controlled microinjection: an in vivo complement for in vitro sensory studies in insects. J. Neurosci. Methods 201, 385-389.

Olsson, S. B., Kuebler, L. S., Veit, D., Steck, K., Schmidt, A., Knaden, M., et al. (2011). A novel multicomponent stimulus device for use in olfactory experiments. J. Neurosci. Methods 195, 1-9.

Pellegrino, M., Nakagawa, T., and Vosshall, L. B. (2010). Single sensillum recordings in the insects Drosophila melanogaster and Anopheles gambiae. J. Vis. Exp. 17, $1-5$.
Peñalva-Arana, D. C., Lynch, M., and Robertson, H. M. (2009). The chemoreceptor genes of the waterflea Daphnia pulex: many GRs but no ORs. BMC Evol. Biol. 9:79. doi: 10.1186/1471-2148-9-79

Robertson, H. M., Warr, C. G., and Carlson, J. R. (2003) Molecular evolution of the insect chemoreceptor gene superfamily in Drosophila melanogaster. Proc. Natl. Acad. Sci. U.S.A. 100, 14537-14542.

Sargsyan, V., Getahun, M. N., Llanos. S. L., Olsson, S. B., Hansson, B. S., and Wicher, D. (2011). Phosphorylation via $\mathrm{PKC}$ regulates the function of the Drosophila odorant coreceptor. Front. Cell. Neurosci. 5:5. doi: 10.3389/fncel.2011.00005

Sato, K., Pellegrino, M., Nakagawa, T., Nakagawa, T., Vosshall, L. B., and Touhara, K. (2008). Insect olfactory receptors are heteromeric ligandgated ion channels. Nature 452, 1002-1006.

Sato, K., Tanaka, K., and Touhara, K. (2011). Sugar-regulated cation channel formed by an insect gustatory receptor. Proc. Natl. Acad. Sci. U.S.A. 108, 11680-11685.

Silbering, A. F., Rytz, R., Grosjean, Y., Abuin, L., Ramdya, P., Jefferis, G. S., et al. (2011). Complementary function and integrated wiring of the evolutionarily distinct Drosophila olfactory subsystems. J. Neurosci. 31, 13357-13375.

Song, E., de Bivort, B., Dan, C., and Kunes, S. (2012). Determinants of the Drosophila odorant receptor pattern. Dev. Cell 22, 363-376.
Su, C. Y., Martelli, C., Emonet, T., and Carlson, J. R. (2011). Temporal coding of odor mixtures in an olfactory receptor neuron. Proc. Natl. Acad. Sci. U.S.A. 108 , 5075-5080.

Theodoni, P., Kovacs, G., Greenlee, M. W., and Deco, G. (2011). Neuronal adaptation effects in decision making. J. Neurosci. 31, 234-246.

Thorne, N., Chromey, C., Bray, S., and Amrein, H. (2004). Taste perception and coding in Drosophila. Curr. Biol. 14, 1065-1079.

Torre, V., Ashmore, J. F., Lamb T. D., and Menini, A. (1995). Transduction and adaptation in sensory receptor cells. J. Neurosci. 75, 7757-7768.

Vickers, N. J., Christensen, T. A., Baker, T. C., and Hildebrand, J. G. (2001) Odour-plume dynamics influence the brain's olfactory code. Nature 410, 466-470.

Vosshall, L. B., Amrein, H., Morozov, P. S., Rzhetsky, A., and Axel, R. (1999). A Spatial map of olfactory receptor expression in the Drosophila antenna. Cell 96, 725-736.

Wang, Z., Singhvi, A., Kong, P., and Scott, K. (2004). Taste representations in the Drosophila brain. Cell 117, 981-991.

Wicher, D., Schafer, R., Bauernfeind, R., Stensmyr, M. C., Heller, R., Heinemann, S. H., et al. (2008). Drosophila odorant receptors are both ligand-gated and cyclic-nucleotide-activated cation channels. Nature 452, 1007-1011.

Yao, C. A., and Carlson, J. R. (2010). Role of G-Proteins in odorsensing and $\mathrm{CO}_{2}$-sensing neurons in Drosophila. J. Neurosci. 30, 4562-4572.

Yao, C. A., Ignell, R., and Carlson, J. R. (2005). Chemosensory coding by neurons in the coeloconic sensilla of the Drosophila antenna. J. Neurosci. 37, 8359-8367.

zur Lage, P. I., Prentice, D. R., Holohan, E. E., and Jarman, A. P. (2003) The Drosophila proneural gene amos promotes olfactory sensillum formation and suppresses bristle formation. Development 130, 4683-4693.

Conflict of Interest Statement: The authors declare that the research was conducted in the absence of any commercial or financial relationships that could be construed as a potential conflict of interest.

Received: 02 August 2012; paper pending published: 06 September 2012; accepted: 28 October 2012; published online: 16 November 2012.

Citation: Getahun MN, Wicher D, Hansson BS and Olsson SB (2012) Temporal response dynamics of Drosophila olfactory sensory neurons depends on receptor type and response polarity. Front. Cell. Neurosci. 6:54. doi: 10.3389/fncel.2012.00054

Copyright (C) 2012 Getahun, Wicher, Hansson and Olsson. This is an openaccess article distributed under the terms of the Creative Commons Attribution License, which permits use, distribution and reproduction in other forums, provided the original authors and source are credited and subject to any copyright notices concerning any third-party graphics etc. 